\title{
Set-membership EIV identification through LMI relaxation techniques
}

\author{
V. Cerone, D. Piga, D. Regruto
}

\begin{abstract}
In this paper the Set-membership Error-InVariables (EIV) identification problem is considered, that is the identification of linear dynamic systems when both the output and the input measurements are corrupted by bounded noise. A new approach for the computation of the Parameters Uncertainty Intervals (PUIs) is discussed. First the problem is formulated in terms of non-convex semi-algebraic optimization. Then, a Linear-Matrix-Inequalities relaxation technique is presented to compute parameters bounds by means of convex optimization. Finally, convergence properties and computational complexity of the given algorithms are discussed. Advantages of the proposed technique with respect to previously published ones are discussed both theoretically and by means of a simulated example.
\end{abstract}

Index Terms-Set-membership identification, errors-invariables, LMI relaxation.

\section{INTRODUCTION}

Linear systems identification has been the subject of extensive studies over the last decades. A good deal of well assessed methodologies for the solution of modeling problems in the time or in the frequency domain through either recursive or batch scheme is presented in [1], [2], [3]. Most of the proposed identification methods rely on the assumption that the input signal is exactly known. Either the output error or the equation error structures are usually considered. In the output error framework only the output measurements are affected by additive noise, while a single error term, added to the difference equation, is used to generically take into account all the possible sources of uncertainty when the equation error structure is considered. However, in many practical problems, input and output data sequences are experimentally collected and, as a consequence, the assumption of noise free input is not a realistic one in such situations. Identification problems where both the input and the output are affected by noise are referred to as errors-in-variables (EIV) problems. Although EIV estimation for static models can be traced back to the seminal works of Adcock [4], [5], identification of dynamic models when all the variables are corrupted by noise still remains a challenging problem and significant research efforts have been devoted to its solution in recent years. A detailed review of the main contributions on identification of linear dynamic systems when both the input and the output are corrupted by stochastic measurement noise, can be found in the recent survey paper by Söderström [6]. A possible alternative to the stochastic description of measurement noise is the bounded error characterization

The authors are with the Dipartimento di Automatica e Informatica, Politecnico di Torino, corso Duca degli Abruzzi 24, 10129 Torino, Italy; e-mail: vito.cerone@polito.it, dario.piga@polito.it, diego.regruto@polito.it; Tel: +39-011-564 7064; Fax: +39-011-564 7198 in the Set-membership identification framework. This is a suitable description when uncertainties are known to belong to a given set: mechanical tolerances, analog-to-digital converter quantization errors, systematic and class errors in measurement equipments are only some examples. In this context, all parameters consistent with the measurements, the error bounds and the assumed model structure, are feasible solutions of the identification problem. The interested reader can find further details on this approach in a number of survey papers (see, e.g., [7], [8]), in the book edited by Milanese et al. [9], and the special issues edited by Norton [10], [11]. On the contrary few works on identification of linear systems when both the input and the output are corrupted by bounded noise can be found in the literature. First insights on the problem were given by Norton [12] in the context of ARMAX models identification with bounded measurement errors. A detailed analysis of the problem is then presented by Veres and Norton in [13] where it is shown that the exact feasible parameter region for dynamic EIV models is described by nonlinear nonconvex bounds, whose shape may become fairly complex when the number of data increases. As a consequence, parameters bounds cannot be easily computed and the use of either polytopic or ellipsoidal outer approximation is suggested. As far as EIV for static models is considered, an exact mathematical description of the feasible parameter set is provided in [14] where relevant topological features, such as convexity and connectedness, are also discussed. Results from [14] are then applied to EIV identification of linear dynamic systems in the work [15] where an outer approximation of the true nonconvex feasible parameter set is obtained as union of a number of polytopes. Such an outer-bounding set is then used to compute the parameter uncertainty intervals (PUI) through the solution of a number of linear programming problems. Actually, such PUIs are not tight and the degree of conservativeness of the approach given in [15] is, in general, not easy to quantify. In this paper we present an alternative approach, based on LMI relaxation techniques, for the computation of parameter uncertainty intervals of EIV dynamics models. The note is organized as follows. Section II is devoted to the formulation of the problem. Relaxation techniques for set-membership EIV identification of linear dynamics systems are presented in Section III. First, the approach given in [15] is briefly reviewed, then, a new LMI relaxation-based technique is presented together with a detailed analysis of its properties. In Section IV, a detailed theoretical comparison between the two relaxation methods is provided, while a simulated example is reported in Section V. Concluding remarks end the paper. 


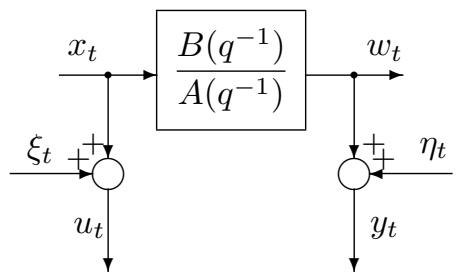

Fig. 1. Errors-in-variable basic setup for dynamic linear system.

\section{PROBLEM FORMULATION}

Consider the single-input single-output (SISO) lineartime-invariant (LTI) system depicted in Fig. 1. The noisefree input sequence is denoted as $x_{t}$ and the linear block is modeled by a discrete time system which transforms $x_{t}$ into the noise-free output $w_{t}$ according to the difference equation

$$
A\left(q^{-1}\right) w_{t}=B\left(q^{-1}\right) x_{t},
$$

where $A(\cdot)$ and $B(\cdot)$ are polynomials in the backward shift operator $q^{-1}\left(q^{-1} w_{t}=w_{t-1}\right)$

$$
\begin{aligned}
& A\left(q^{-1}\right)=1+a_{1} q^{-1}+\ldots+a_{n a} q^{-n a} \\
& B\left(q^{-1}\right)=b_{0}+b_{1} q^{-1}+\ldots+b_{n b} q^{-n b} .
\end{aligned}
$$

Both input and output data sequences are corrupted by additive noise, $\xi_{t}$ and $\eta_{t}$ respectively:

$$
\begin{aligned}
& u_{t}=x_{t}+\xi_{t}, \\
& y_{t}=w_{t}+\eta_{t},
\end{aligned}
$$

where $\xi_{t}$ and $\eta_{t}$ are assumed to range within given bounds $\Delta \xi_{t}$ and $\Delta \eta_{t}$ respectively, that is

$$
\begin{aligned}
\left|\xi_{t}\right| & \leq \Delta \xi_{t} \\
\left|\eta_{t}\right| & \leq \Delta \eta_{t} .
\end{aligned}
$$

The unknown parameter vector $\theta \in \mathbb{R}^{p}$ to be identified is defined as:

$$
\theta=\left[\begin{array}{lllllll}
a_{1} & \ldots & a_{n a} & b_{0} & b_{1} & \ldots & b_{n b}
\end{array}\right]^{\mathrm{T}},
$$

where $n a+n b+1=p$, while the feasible parameter set (FPS) $\mathcal{D}_{\theta}$ is defined as:

$$
\begin{gathered}
\mathcal{D}_{\theta}=\left\{\theta \in \mathbb{R}^{p}: A\left(q^{-1}\right)\left(y_{t}-\eta_{t}\right)=B\left(q^{-1}\right)\left(u_{t}-\xi_{t}\right),\right. \\
\left.\left|\xi_{t}\right| \leq \Delta \xi_{t}, \quad\left|\eta_{t}\right| \leq \Delta \eta_{t} ; t=1, \ldots, N\right\}
\end{gathered}
$$

where $N$ is the length of data sequences. Equation (9) provides an exact description of the set of all possible values of the unknown parameter $\theta$ consistent with measured data, error bounds and assumed model structure. In this work we address the problem of the evaluation of parameter uncertainty intervals defined as

$$
P U I_{j}=\left[\underline{\theta}_{j}, \bar{\theta}_{j}\right]
$$

where

$$
\begin{aligned}
& \underline{\theta}_{j}=\min _{\theta \in \mathcal{D}_{\theta}} \theta_{j}, \\
& \bar{\theta}_{j}=\max _{\theta \in \mathcal{D}_{\theta}} \theta_{j} .
\end{aligned}
$$

The computation of $P U I_{j} \mathrm{~s}$ requires the solution of constrained optimization problems (11) and (12). Since $\mathcal{D}_{\theta}$ is a non-convex set defined by nonlinear constraints (see, e.g., [13]), standard nonlinear optimization tools (gradient method, Newton method, etc.) cannot be used since they can trap in local minima which may result arbitrary far from the global one. Thus, the $P U I_{j}$ s obtained using such tools are not guaranteed to contain the true unknown parameter, which is a key requirement of any set-membership identification method. One possible solution to overcome this problem is to relax (11) and (12) to convex problems to obtain a lower (upper) bound of $\underline{\theta}_{j}\left(\bar{\theta}_{j}\right)$.

The relaxation technique presented in [15], which consists in the application of the results for static EIV problems derived in [14], provides an outer approximation of the FPS $\mathcal{D}_{\theta}$. In this work, we present a new approach to compute the PUIs through LMI relaxation techniques.

\section{RELAXATION TECHNIQUES FOR EIV PROBLEMS}

The static EIV relaxation procedure described in [14], [15] is briefly reviewed in section III-A for completeness and self-consistency of the paper. Then, the new technique to evaluate parameters uncertainty intervals through LMI relaxation techniques is presented in section III-B.

\section{A. Static EIV relaxation procedure}

The main idea in [14] is to relax the original problems (11)-(12) assuming that uncertain variables $\xi_{t}$ and $\eta_{t}$ in (9) vary independently. In other words, such a relaxation technique is equivalent to assume that output and input samples are collected from $N$ independent measurements. In this way, an outer approximation $\mathcal{D}_{\theta}^{s}$ of $\mathcal{D}_{\theta}$ can be obtained. Its mathematical description is given by the following result.

\section{Result 1 - Description of the FPS for a static EIV problem [14]}

A necessary and sufficient condition for $\theta$ to belong to the set $\mathcal{D}_{\theta}^{s}$ when uncertain variables vary independently is:

$$
\begin{aligned}
& \left(\phi_{t}-\Delta \phi_{t}\right) \theta \leq y_{t}+\Delta \eta_{t}, \\
& \left(\phi_{t}+\Delta \phi_{t}\right) \theta \geq y_{t}-\Delta \eta_{t} \quad t=1, \ldots, N
\end{aligned}
$$

where

$$
\begin{aligned}
\Delta \phi_{t}= & {\left[\Delta \eta_{t-1} \operatorname{sgn}\left(a_{1}\right) \ldots \Delta \eta_{t-n a} \operatorname{sgn}\left(a_{n a}\right)\right.} \\
& \left.\Delta \xi_{t} \operatorname{sgn}\left(b_{0}\right) \Delta \xi_{t-1} \operatorname{sgn}\left(b_{1}\right) \ldots \Delta \xi_{t-n b} \operatorname{sgn}\left(b_{n b}\right)\right]^{\mathrm{T}}
\end{aligned}
$$

Therefore, the FPS $\mathcal{D}_{\theta}^{s}$ for a static EIV problem has the form:

$$
\begin{aligned}
\mathcal{D}_{\theta}^{s}= & \left\{\theta \in \mathbb{R}^{p}:\left(\phi_{t}-\Delta \phi_{t}\right) \theta \leq y_{t}+\Delta \eta_{t},\right. \\
& \left.\left(\phi_{t}+\Delta \phi_{t}\right) \theta \geq y_{t}-\Delta \eta_{t} ; \quad t=1, \ldots, N .\right\}
\end{aligned}
$$


It must be pointed out that $\mathcal{D}_{\theta}^{s}$ is an outer approximation of $\mathcal{D}_{\theta}$, i.e. $\mathcal{D}_{\theta}^{s} \supseteq \mathcal{D}_{\theta}$.

The set $\mathcal{D}_{\theta}^{s}$ is defined by piecewise linear constraints and, although generally non-convex, it is the union of at most $2^{p}$ convex set $\mathcal{D}_{\theta i}^{s}$, that is:

$$
\mathcal{D}_{\theta}^{s}=\bigcup_{i=1}^{2^{p}} \mathcal{D}_{\theta i}^{s}
$$

where $\mathcal{D}_{\theta i}^{s}$ is defined by $2 N+p$ linear constraints and it is the intersection between $\mathcal{D}_{\theta i}^{s}$ and the i-th orthant of the space $\mathbb{R}^{p}$. Let the relaxed parameter uncertainty interval $P U I_{j}$ be:

$$
P U I_{j}^{s}=\left[\underline{\theta}_{j}^{s}, \bar{\theta}_{j}^{s}\right]
$$

where:

$$
\begin{aligned}
\underline{\theta}_{j}^{s} & =\min _{i=1, \ldots, 2^{p}} \underline{\theta}_{j i}^{s} \\
\bar{\theta}_{j}^{s} & =\max _{i=1, \ldots, 2^{p}} \bar{\theta}_{j i}^{s}
\end{aligned}
$$

and

$$
\begin{aligned}
\underline{\theta}_{j i}^{s} & =\min _{\theta \in \mathcal{D}_{\theta i}^{s}} \theta_{j} \\
\bar{\theta}_{j i}^{s} & =\max _{\theta \in \mathcal{D}_{\theta i}^{s}} \theta_{j}
\end{aligned}
$$

Note that, since $\mathcal{D}_{\theta}^{s}$ is an outer approximation of $\mathcal{D}_{\theta}, \underline{\theta}_{j}^{s} \leq \underline{\theta}_{j}$ and $\bar{\theta}_{j}^{s} \geq \bar{\theta}_{j}$, therefore

$$
P U I_{j}^{s} \supseteq P U I_{j} \quad j=1, \ldots, p .
$$

Unfortunately, static EIV does not guarantee that relaxed $P U I_{j}^{s}$ converges to the tight $P U I_{j}$, even if the number of measurements $N$ goes to infinity.

\section{Computational complexity}

The definition of $P U I_{j}^{s}$ for all $j=1, \ldots, p$ requires the computation of $\underline{\theta}_{j}^{s}$ and $\bar{\theta}_{j}^{s}$, which in turn requires the solution of $2 p 2^{p}$ Linear Programming (LP) optimization problems, since problems (18)-(19) must be solved for each of the $p$ parameters $\theta_{j}$ and in each of the $2^{p}$ set $D_{\theta i}^{s}$.

\section{B. Dynamic EIV through LMI relaxation}

In this subsection the computation of the approximate parameter uncertainty interval $P U I_{j}^{d}$ through LMI relaxation techniques is described. The following result shows as (11) and (12) can be rewritten as constrained polynomial optimization problems. In order to comply with the conference page limit constraint, the proof of all the following results and properties are omitted and can be found in [16].

\section{Result 2 - Computation of $\underline{\theta}_{j}$ and $\bar{\theta}_{j}$}

$\underline{\theta}_{j}$ and $\bar{\theta}_{j}$ can be computed solving the following optimization problems:

$$
\begin{aligned}
& \underline{\theta}_{j}=\min _{\theta, \xi, \eta} \theta_{j} \\
& \text { s.t. } \\
& \left\{\begin{array}{l}
y_{t}=-\sum_{i=1}^{n a}\left(y_{t-i}-\eta_{t-i}\right) a_{i}+\sum_{j=0}^{n b}\left(u_{t-j}-\xi_{t-j}\right) b_{j}+\eta_{t} \\
\xi_{t} \leq \Delta \xi_{t}, \quad \xi_{t} \geq-\Delta \xi_{t}, \quad \eta_{t} \leq \Delta \eta_{t}, \quad \eta_{t} \geq-\Delta \eta_{t}, \quad t=1, \ldots, N
\end{array}\right.
\end{aligned}
$$

$$
\begin{aligned}
& \bar{\theta}_{j}=\max _{\theta, \xi, \eta} \theta_{j} \\
& \text { s.t. } \\
& \quad\left\{\begin{array}{l}
y_{t}=-\sum_{i=1}^{n a}\left(y_{t-i}-\eta_{t-i}\right) a_{i}+\sum_{j=0}^{n b}\left(u_{t-j}-\xi_{t-j}\right) b_{j}+\eta_{t} \\
\xi_{t} \leq \Delta \xi_{t}, \quad \xi_{t} \geq-\Delta \xi_{t}, \quad \eta_{t} \leq-\Delta \eta_{t}, \quad t=1, \ldots, N
\end{array}\right.
\end{aligned}
$$

where $\eta=\left[\eta_{1}, \quad \eta_{2}, \ldots, \eta_{N}\right]^{\mathrm{T}}$ and $\xi=\left[\begin{array}{ll}\xi_{1}, & \xi_{2}, \ldots, \xi_{N}\end{array}\right]^{\mathrm{T}}$.

Remark 1 - The computation of $\underline{\theta}_{j}$ and $\bar{\theta}_{j}$ is then reduced to a minimization (maximization) problem over $p+2 N$ optimization variables. The feasible set is semialgebraic, defined by $N$ bilinear polynomial equality constraints and $4 N$ linear inequalities. It must be noted that an equality constraint can be written as a set of two inequality constraints. For instance, the equality constraints $x=0$ can be written as $x \geq 0$ and $x \leq 0$. Therefore, the $N$ equality constraints in problems (21)-(22) can be written as a set of $2 N$ inequality constraints. Therefore, the total number of inequality constraints in (21)-(22) is $6 \mathrm{~N}$.

Considerable efforts have been devoted in the last years to approximate semi-algebraic optimization problems by a hierarchy of convex LMI relaxations (see the survey paper [17] for a review of the literature on the subject). In particular, the approach proposed in [18] is based on the representation of nonnegative polynomials as Sum of Squares (SOS), while the dual theory of moments is exploited in [19]. More specifically, the relaxation technique described in [19] solves semidefinite programming problems, whose optima are guaranteed to converge monotonically to the global optima of the original optimization problem as the length of the number of successive LMI relaxations, the relaxation order $\delta$, increases. Application of the method presented in [19] to problems (21) and (22), for a given order of relaxation $\delta$ leads to the computation of the $\delta$-relaxed bounds as

$$
\begin{aligned}
& \underline{\theta}_{j}^{d}(\delta)=\min _{x \in \mathcal{D}_{x}^{d \delta}} f_{j}(x), \\
& \bar{\theta}_{j}^{d}(\delta)=\max _{x \in \mathcal{D}_{x}^{d \delta}} f_{j}(x),
\end{aligned}
$$


where $x$ is the vector variables, called LMI decision variables. The function $f_{j}(x)$ is linear and $\mathcal{D}_{x}^{d \delta}$ is a convex set defined by linear matrix inequalities.

\section{Convergency properties}

Let us define the relaxed uncertainty interval $P U I_{j}^{d}(\delta)$ for a relaxation order $\delta$ as:

$$
P U I_{j}^{d}(\delta)=\left[\underline{\theta}_{j}^{d}(\delta), \bar{\theta}_{j}^{d}(\delta)\right]
$$

then the following result holds.

\section{Result 3 - Monotone convergence to tight parameter} uncertainty intervals

The relaxed uncertainty interval $P U I_{j}^{d}(\delta)$ becomes tighter as $\delta$ increases, that is:

$$
\begin{gathered}
\underline{\theta}_{j}^{d}(\delta) \leq \underline{\theta}_{j}^{d}(\delta+1) \leq \underline{\theta}_{j} \text { for } \delta=1,2, \ldots \\
\bar{\theta}_{j}^{d}(\delta) \geq \bar{\theta}_{j}^{d}(\delta+1) \geq \bar{\theta}_{j} \text { for } \delta=1,2, \ldots
\end{gathered}
$$

Thus, relaxed parameter uncertainty intervals $P U I_{j}^{d}(\delta)$ converge to the tight uncertainty intervals $P U I_{j}$, as the LMI relaxation order goes to infinity, that is:

$$
\begin{aligned}
& \lim _{\delta \rightarrow \infty} \underline{\theta}_{j}^{d}(\delta)=\underline{\theta}_{j} \\
& \lim _{\delta \rightarrow \infty} \bar{\theta}_{j}^{d}(\delta)=\bar{\theta}_{j}
\end{aligned}
$$

It must be pointed out that the number of LMI decision variables $x$ and the size of matrix that describes $\mathcal{D}_{x}^{d \delta}$ increases with the relaxation order $\delta$, therefore, there is a tradeoff between accuracy and complexity of the LMI relaxation. Although the convergency is guaranteed as the relaxation order goes to infinity, exact global optima have been obtained in practice for a number of small and medium size problems with a reasonably low relaxation order (see [20] for a collection of test problems solved with relaxation order less or equal to 4 ).

\section{Parameter uncertainty intervals computational complexity}

The evaluation of $P U I_{j}^{d}(\delta)$ requires the solution of the SDP problems (23)-(24) for $j=1, \ldots, p$. The complexity of such problems as a function of the relaxation order $\delta$, the number $p$ of unknown parameters and the number of measurements $N$ is described by the following property.

\section{Property 1 - Complexity of LMI relaxation}

(P1.1) The number of LMI decision variables $x$ is $\left(\begin{array}{c}2 N+p+2 \delta \\ 2 N+p\end{array}\right)$-1, i.e.:

- $O\left(\delta^{p+2 N}\right)$ for fixed $p$ and $N$,

- $O\left(N^{2 \delta}+p^{2 \delta}\right)$ for fixed $\delta$.

(P1.2) The size of LMI is $\left(\begin{array}{c}2 N+p+\delta \\ 2 N+p\end{array}\right)+$ $6 N\left(\begin{array}{c}2 N+p+\delta-1 \\ 2 N+p\end{array}\right)$, i.e.:
- $O\left(N \delta^{(2 N+p)}\right)$ for fixed $p$ and $N$,

- $O\left(N^{\delta}+p^{\delta}\right)$ for fixed $\delta$.

The complexity analysis of Property 1 is performed with reference to the LMI relaxation technique proposed in [19]. Several efforts on the reduction of LMI relaxation complexity, exploiting the particular structure of the optimization problem, have been carried out in the recent years (see for instance [21], [22], [23]). Roughly speaking, a polynomial optimization problem has a structured sparsity when the objective function and each constraint that define the feasible region of the optimization problem involve only a small subset of variables (see [17], [21], [24]). The following result shows that (11)-(12) have a structured sparsity that can be exploited to reduce LMI complexity.

\section{Property 2 - Structure of the original optimization problems}

Problems (11)-(12) enjoy the following features:

(P2.1) The objective function is linear in the variable $\theta_{j}$.

(P2.2) Each linear constraint depend only on the noise $\xi_{t}$ or $\eta_{t}$.

(P2.3) The generic polynomial constraint at time $k$ depends only on $2 p+1$ variables, i.e.:

- the unknown parameters $\theta_{j}, j=1, \ldots, p$

- the output sample noise $\eta_{t}, t=k, k-$ $1, \ldots, k-n a$

- the input sample noise $\xi_{t}, t=$ $k, k-1, \ldots, k-n b$

Since (21)-(22) satisfy Property 2, the LMI relaxation method for problems with structured sparsity in the original data, discussed in [24] in the spirit of the work of Waki et al [21], can be exploited in order to relax the original problems (21)-(22). Such a method enjoys the features reported in the following property.

\section{Property 3 - Reduced complexity of LMI relaxation}

(P3.1) the number of LMI variables $x$ is bounded by $N\left(\begin{array}{c}2 p+1+2 \delta \\ 2 p+1\end{array}\right)$, i.e.:

- $O\left(\delta^{2 p+1}\right)$ for fixed $p$ and $N$,

- $O\left(N p^{2 \delta}\right)$ for fixed $\delta$.

(P3.2) There are $N$ LMI constraints of size $\left(\begin{array}{c}2 p+1+\delta \\ 2 p+1\end{array}\right)$ and $6 N \quad$ LMI constraints, whose largest size is $\left(\begin{array}{c}2 p+1+\delta-1 \\ 2 p+1\end{array}\right)$, then the size of LMI is:

- $O\left(N \delta^{(2 p+1)}\right)$ for fixed $p$,

- $O\left(N p^{\delta-1}\right)$ for fixed $\delta$.

An efficient implementation of LMI relaxation for polynomial optimization problems with structured sparsity has been developed by Waki, Kim, Kojima and Muramatsu in the Matlab package SparsePOP which exploits the LMI 
solver SeDuMi [25] to solve SDP problems in polynomial time. It must be noted that, in general, such a relaxation method does not guarantee convergency to the global optimum of the original constrained polynomial problem. However, it is shown in [24] that if the structure of the original polynomial problem satisfies suitable assumptions on the sparsity structure, this LMI relaxation method provides a solution that converges to the global optimum of the original polynomial optimization problem, as the relaxation order $\delta$ goes to infinity. The following result describes the convergency properties of the $P U I_{j}^{d}(\delta)$ to the global optimum $P U I_{j}$ when LMI relaxation for problems with structured sparsity is exploited.

Result 4 - The original optimization problems (21)(22) satisfy the sparsity conditions given by Lasserre in [24]. Therefore, the resulting sequence of relaxed problems, obtained applying the LMI relaxation method for problems with structured sparsity, converges to the global optima of problems (21)-(22), as far as $\delta$ goes to infinity, which means that:

$$
\lim _{\delta \rightarrow \infty} P U I_{j}^{d}(\delta)=P U I_{j} .
$$

Remark 2 - The relaxation procedure presented in this section allows the computation of the relaxed $P U I_{j}^{d}$ exploiting the LMI relaxation technique in order to approximate the tight $P U I_{j}$. While in the static EIV the sample noise sequences are assumed to be not correlated, in the dynamic EIV through LMI relaxation the sample noise $\xi_{t}$ and $\eta_{t}$ are treated as optimization variables of the original problems (21)-(22), in order to preserve the correlation between consecutive measurements.

\section{COMPARISON BETWEEN RELAXATION TECHNIQUES}

In this section a comparison between static EIV approach and dynamic EIV through LMI relaxation techniques is presented. Furthermore, we suggest how the two techniques can be combined in order to improve the estimation of the parameter bounds. As previously discussed, one of the main advantage of the LMI relaxation over the static EIV relaxation is the convergency of the relaxed $P U I_{j}^{d}$ to the tight $P U I_{j}$, as stated in results 3 and 4 . Therefore, as the relaxation order goes to infinity, dynamic EIV through LMI relaxation provides tighter parameters bounds than the static EIV approach, that is:

$$
\lim _{\delta \rightarrow \infty} P U I_{j}^{d}(\delta)=P U I_{j} \subseteq P U I_{j}^{s}, \quad j=1, \ldots, p .
$$

However, for a finite value of the relaxation order $\delta$ there is no guarantee that $P U I_{j}^{d}(\delta)$ is tighter than $P U I_{j}^{s}$. In order to obtain parameters bounds tighter than $P U I_{j}^{s}$ independently on relaxation order $\delta$, the following algorithm is suggested:

\section{Algorithm 1}

(A1.1) For each $j=1, \ldots, p$, compute $\underline{\theta}_{j}^{s}$ and $\bar{\theta}_{j}^{s}$ exploiting static EIV relaxation.
(A1.2) Add the linear constraints: $\underline{\theta}_{j}^{s} \leq \theta_{j}$ and $\theta_{j} \leq \bar{\theta}_{j}^{s}$ for $j=1, \ldots, p$ to problems (21) and (22).

(A1.3) Compute the approximated solution of (21) and (22) (with linear constraints added at point 2), solving the LMI problems (23)-(24) for a relaxation order $\delta$.

Remark 3 - Note that, since the additional constraints imposed in the second step of Algorithm 1 are linear, they are satisfied for any order of relaxation $\delta$ [16]. Therefore, if Algorithm 1 is applied to compute $P U I_{j}^{d}(\delta)$, it follows that:

$$
P U I_{j}^{d}(\delta) \subseteq P U I_{j}^{s} \begin{aligned}
& j=1, \ldots, p \\
& \delta=1,2, \ldots
\end{aligned}
$$

The main idea of this procedure is to compute the parameter bounds using, first, the static relaxation in order to obtain initial outer bounds $P U I_{j}^{s}$ of the intervals $P U I_{j}$. Then, the $P U I_{j}^{d}(\delta)$ are computed solving the constrained polynomial problems (21)-(22) with the additional constraints that the parameter $\theta_{j}$ has to be contained into the interval $P U I_{j}^{s}$.

\section{A simulated eXAmple}

In this section we illustrate the presented parameter bounding procedure through a numerical example. The parameter uncertainty intervals are computed exploiting both static EIV approach and dynamic EIV through LMI relaxation. The system considered here is characterized by (1), (2) and (3) with: $A\left(q^{-1}\right)=\left(1+1.15 q^{-1}+0.8 q^{-2}\right)$ and $B\left(q^{-1}\right)=$ $\left(-2.45 q^{-1}+2.1 q^{-2}\right)$. Thus, the true parameter vector is $\theta^{\mathrm{T}}=\left[\begin{array}{llll}a_{1} & a_{2} & b_{1} & b_{2}\end{array}\right]=\left[\begin{array}{llll}1.15 & 0.8 & -2.45 & 2.1\end{array}\right]$. The input is a random sequence uniformly distributed between $[-1,+1]$. Both input and output sequence are corrupted by random additive noise, uniformly distributed between $\left[-\Delta \xi_{t},+\Delta \xi_{t}\right]$ and $\left[-\Delta \eta_{t},+\Delta \eta_{t}\right]$, respectively. The chosen error bounds $\Delta \xi_{t}$ and $\Delta \eta_{t}$ are such that the Signal to Noise Ratios on the input $S N R_{x}$ and on the output $S N R_{w}$, defined as:

$$
\begin{aligned}
& S N R_{x}=10 \log \left\{\sum_{t=1}^{N} x_{t}^{2} / \sum_{t=1}^{N} \xi_{t}^{2}\right\}, \\
& S N R_{w}=10 \log \left\{\sum_{t=1}^{N} w_{t}^{2} / \sum_{t=1}^{N} \eta_{t}^{2}\right\}
\end{aligned}
$$

are $25 \mathrm{db}$ and $31 \mathrm{db}$, respectively. The number of measurements $N$ exploited to compute the parameters bounds is 300 . $\underline{\theta}_{j}^{s}$ and $\bar{\theta}_{j}^{s}$ that define the $P U I_{j}^{s}$ relaxing original problems (11)-(12) by means of static EIV approach are first evaluated. The obtained results are reported in Table II, which reports the center $\theta_{j}^{c s}$ and the parameter uncertainty bounds $\Delta \theta_{j}^{s}$, defined as:

$$
\theta_{j}^{c s}=\frac{\bar{\theta}_{j}^{s}+\underline{\theta}_{j}^{s}}{2}, \quad \Delta \theta_{j}^{s}=\frac{\bar{\theta}_{j}^{s}-\underline{\theta}_{j}^{s}}{2}
$$

Then, dynamic EIV thorugh LMI relaxation is exploited, with a relaxation order $\delta=2$. Table III reports the obtained 
values of $\underline{\theta}_{j}^{d}(2)$ and $\bar{\theta}_{j}^{d}(2)$, the center $\theta_{j}^{c d}(2)$ and the parameter uncertainty bounds $\Delta \theta_{j}^{d}(2)$, which are defined as:

$$
\theta_{j}^{c d}(2)=\frac{\bar{\theta}_{j}^{d}(2)+\underline{\theta}_{j}^{d}(2)}{2}, \quad \Delta \theta_{j}^{d}(2)=\frac{\bar{\theta}_{j}^{d}(2)-\underline{\theta}_{j}^{d}(2)}{2} .
$$

Table II: Static EIV relaxation - Parameter central estimates $\left(\theta_{j}^{c s}\right)$, parameter bounds $\left(\underline{\theta}_{j}^{s}\right.$, $\begin{array}{ccccc}\bar{\theta}_{j}^{s} \text { ) and } & \text { parameter uncertainty bounds } \Delta \theta_{j}^{s} \\ \text { True } & \underline{\theta}^{s} & \theta_{j}^{c s} & \bar{\theta}_{j}^{s}\end{array}$

\begin{tabular}{ccccc}
$\begin{array}{c}\text { True } \\
\text { Value }\end{array}$ & $\underline{\theta}_{j}^{s}$ & $\theta_{j}^{c s}$ & $\theta_{j}$ & $\Delta \theta_{j}^{s}$ \\
\hline 1.1500 & 0.9689 & 1.1583 & 1.3477 & 0.1894 \\
0.8000 & 0.6319 & 0.8258 & 1.0197 & 0.1939 \\
-2.4500 & -5.0594 & -2.7386 & -0.4178 & 2.3208 \\
2.1000 & 0.1470 & 2.4363 & 4.7256 & 2.2893 \\
\hline
\end{tabular}

Table III: Dynamic EIV through LMI relaxation - Parameter central estimates $\left(\theta_{j}^{c d}(2)\right)$,

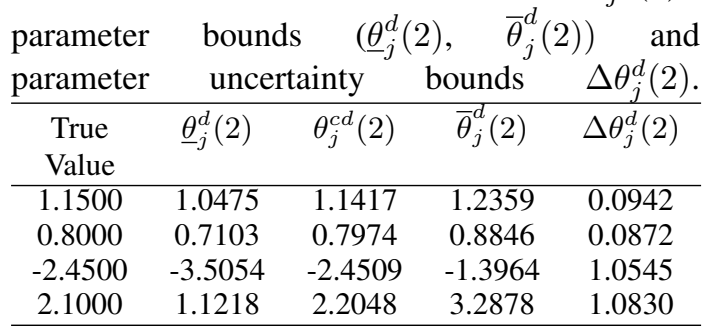

A comparison between the results reported in Table II and Table III shows that, although a low relaxation order $\delta$ has been used in the dynamic EIV LMI relaxation technique, the uncertainty bounds $\Delta \theta_{j}^{d}(2)$ are less than half the uncertainty bounds $\Delta \theta_{j}^{s}$, for each parameter $\theta_{j}$.

\section{CONCLUSIONS}

A procedure to evaluate the parameters bounds of a linear dynamic system for Set-Membership EIV problems is presented. The parameters bounds evaluation is formulated as a collection of constrained polynomial optimization problems, whose global optima is approximated by means of a hierarchy of convex SDP relaxed problems, which guarantees monotone convergence to global optima as the relaxation order increases. The particular structure of the original optimization problems makes it possible a reduction of the computational complexity of the SDP relaxed problems, preserving the convergence to the global optima. Finally, the capabilities of such LMI relaxation technique to provide a less conservative estimation of the parameter bounds with respect to the static EIV is shown both theoretically and by means of a numerical example.

\section{REFERENCES}

[1] L. Ljung, System Identification, Theory for the User. Upper Saddle River: Prentince Hall, 1999.

[2] T. Söderström and P. Stoica, System Identification. Upper Saddle River: Prentice Hall, 1989.

[3] M. Verhaegen and V. Verdult, Filtering and System Identification, A Least Squares Approach. Cambridge University Press, 2007.
[4] R. Adcock, "Note on the methods of least squares," The Analyst, vol. 4, no. 6, pp. 183-184, 1877.

[5] _ , "A problem in least squares," The Analyst, vol. 5, no. 2, pp. $53-54,1878$.

[6] T. Söderström, "Errors-in-variables methods in system identification," Automatica, vol. 43, no. 6, pp. 939-958, 2007.

[7] M. Milanese and A. Vicino, "Optimal estimation theory for dynamic sistems with set membership uncertainty: an overview," Automatica, vol. 27(6), pp. 997-1009, 1991.

[8] E. Walter and H. Piet-Lahanier, "Estimation of parameter bounds from bounded-error data: a survey," Mathematics and Computers in simulation, vol. 32, pp. 449-468, 1990.

[9] M. Milanese, J. Norton, H. Piet-Lahanier, and E. Walter, Eds., Bounding approaches to system identification. New York: Plenum Press, 1996.

[10] J. Norton (Ed.), "Special issue on bounded-error estimation," Int. J. of Adapt. Control \& Sign. Proces., vol. 8, no. 1, 1994.

[11] _ - "Special issue on bounded-error estimation," Int. J. of Adapt. Control \& Sign. Proces., vol. 9, no. 1, 1995.

[12] J. Norton, "Identification of parameter-bounds for armax models from records with bounded noise," International Journal of Control, vol. 45, pp. 375-390, 1987.

[13] S. Veres and J. Norton, "Parameter-bounding algorithms for linear errors in variables models," in Proc. of IFAC/IFORS Symposium on Identification and System Parameter Estimation, 1991, pp. 1038-1043.

[14] V. Cerone, "Feasible parameter set for linear models with bounded errors in all variable," Automatica, vol. 29, no. 6, pp. 1551-1555, 1993

[15] _ _ "Parameter bounds for armax models from records with bounded errors in variables," Int. J. Control, vol. 57, no. 1, pp. 225-235, 1993.

[16] V. Cerone, D. Piga, and D. Regruto, "Parameters bounds evaluation for set-membership eiv problems through lmi relaxation techniques,' DAUIN Internal Report 2009/09/14, 2009.

[17] M. Laurent, "Sums of squares, moment matrices and optimization over polynomials," Emerging Applications of Algebraic Geometry, Vol. 149 of IMA Volumes in Mathematics and its Applications, M. Putinar and S. Sullivant (eds.), pp. 157-270, 2009.

[18] P. Parrillo, "Semidefinite programming relaxations for semialgebraic problems," Mathematical Programming, vol. 96, pp. 293-320, 2003.

[19] J. B. Lasserre, "Global optimization with polynomials and the problem of moments," SIAM Journal on Optimization, vol. 11, pp. 796-817, 2001

[20] D. Henrion and J. B. Lasserre, "Solving nonconvex optimization problems," IEEE Control Systems Magazine, vol. 24, no. 3, pp. 72-83, 2004.

[21] H. Waki, S. Kim, M. Kojima, and M. Muramatsu, "Sums of squares and semidefinite programming relaxations for polynomial optimization problems with structured sparsity," SIAM Journal on Optimization, vol. 17, no. 1, pp. 218-242, 2006.

[22] M. Kojima, S. Kim, and H. Waki, "Sparsity in sums of squares of polynomials," Mathematical Programming, vol. 103, pp. 45-62, 2005.

[23] P. Parrillo, "Exploiting algebraic structure in sum of squares programs," Positive Polynomials in Control, D. Henrion and A. Garulli (eds.), pp. 181-194, 2005.

[24] J. Lasserre, "Convergent semidefinite relaxations in polynomial optimization with sparsity," SIAM Journal on Optimization, vol. 17, no. 1 , pp. 822-843, 2006.

[25] J. F. Sturm, "Using SeDuMi 1.02, a MATLAB Toolbox for optimization over symmetric cones," Optim. Methods Software, vol. 11, no. 12, pp. 625-653, 1999. 